\title{
Mechanism of gasdermin D recognition by inflammatory caspases and their inhibition by a gasdermin D-derived peptide inhibitor
}

\author{
Jie Yang ${ }^{\mathrm{a}, \mathrm{b}}$, Zhonghua Liu ${ }^{\mathrm{a}}$, Chuanping Wanga, Rui Yang a,c, Joseph K. Rathkey ${ }^{\mathrm{a}}$, Otis W. Pinkard ${ }^{\mathrm{a}}$, Wuxian Shid, \\ Yinghua Chen ${ }^{\mathrm{e}}$, George R. Dubyak ${ }^{\mathrm{a}, \mathrm{f}}$, Derek W. Abbott ${ }^{\mathrm{a}}$, and Tsan Sam Xiao ${ }^{\mathrm{a}, \mathrm{g}, 1}$
}

\begin{abstract}
aDepartment of Pathology, Case Western Reserve University School of Medicine, Cleveland, $\mathrm{OH} 44106$; ${ }^{\mathrm{b}}$ Graduate Program in Physiology and Biophysics, Department of Physiology and Biophysics, Case Western Reserve University School of Medicine, Cleveland, OH 44106; ${ }^{\circ}$ Department of Biology, Case Western Reserve University, Cleveland, $\mathrm{OH} 44106 ;{ }^{d}$ Center for Proteomics and Bioinformatics, Center for Synchrotron Biosciences, Case Western Reserve University

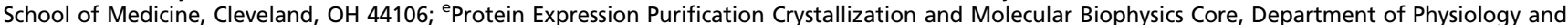
Biophysics, Case Western Reserve University School of Medicine, Cleveland, OH 44106; ' Department of Physiology and Biophysics, Case Western Reserve University School of Medicine, Cleveland, OH 44106; and ${ }^{9}$ Cleveland Center for Membrane and Structural Biology, Case Western Reserve University School of Medicine, Cleveland, $\mathrm{OH} 44106$
\end{abstract}

Edited by Hao Wu, Department of Biological Chemistry and Molecular Pharmacology, Harvard Medical School, Boston, MA, and approved May 22, 2018 (received for review January 10, 2018)

\begin{abstract}
The inflammasomes are signaling platforms that promote the activation of inflammatory caspases such as caspases-1, $-4,-5$, and -11. Recent studies identified gasdermin D (GSDMD) as an effector for pyroptosis downstream of the inflammasome signaling pathways. Cleavage of GSDMD by inflammatory caspases allows its $\mathrm{N}$-terminal domain to associate with membrane lipids and form pores that induce pyroptotic cell death. Despite the important role of GSDMD in pyroptosis, the molecular mechanisms of GSDMD recognition and cleavage by inflammatory caspases that trigger pyroptosis are poorly understood. Here, we demonstrate that the catalytic domains of inflammatory caspases can directly bind to both the full-length GSDMD and its cleavage site peptide, FLTD. A GSDMD-derived inhibitor, $\mathrm{N}$ acetyl-Phe-Leu-Thr-Asp-chloromethylketone (Ac-FLTD-CMK), inhibits GSDMD cleavage by caspases-1, $-4,-5$, and -11 in vitro, suppresses pyroptosis downstream of both canonical and noncanonical inflammasomes, as well as reduces IL-1 $\beta$ release following activation of the NLRP3 inflammasome in macrophages. By contrast, the inhibitor does not target caspase-3 or apoptotic cell death, suggesting that Ac-FLTDCMK is a specific inhibitor for inflammatory caspases. Crystal structure of caspase-1 in complex with AC-FLTD-CMK reveals extensive enzymeinhibitor interactions involving both hydrogen bonds and hydrophobic contacts. Comparison with other caspase-1 structures demonstrates drastic conformational changes at the four active-site loops that assemble the catalytic groove. The present study not only contributes to our understanding of GSDMD recognition by inflammatory caspases but also reports a specific inhibitor for these caspases that can serve as a tool for investigating inflammasome signaling.
\end{abstract}

inflammasome | inflammatory caspases | gasdermin D | pyroptosis | covalent inhibitor

7 he he inflammasomes are signaling platforms that play crucial roles in inflammatory responses to infections and danger signals (1-3). The canonical inflammasome complex is composed of a sensor protein, an adaptor protein called ASC (apoptosisassociated speck-like protein containing a $C A R D$ ), and inflammatory caspases such as caspase-1. Activation of the canonical inflammasomes promotes the maturation of caspase-1, which cleaves both proinflammatory cytokines (4) and gasdermin D (GSDMD) (5-7). GSDMD is suggested to adopt an autoinhibited conformation (through intramolecular domain interactions) (810 ), which is released by caspase cleavage to allow its N-terminal p30 fragment to interact with membrane lipids and to assemble pores that induce pyroptosis, a lytic form of cell death that releases intracellular contents $(8,11-15)$. The GSDMD pores contribute to the release of mature cytokines such as IL-1 $\beta$ and IL-18, which in turn recruit more immune cells to promote inflammation (16). The noncanonical inflammasomes play important roles in immune response to intracellular bacterial infections (17-19). The caspase activation and recruitment domains (CARDs) of caspases-4, -5 , and -11 can directly bind to lipopolysaccharides (LPSs) and mediate the activation of the noncanonical inflammasomes (20). This leads to cleavage of GSDMD and induction of pyroptosis. Similar to GSDMD, the N-terminal domains of other gasdermin family members such as GSDMA/GSDMA3, GSDMB, GSDMC, and GSDME/DFNA5 can induce pyroptosis in mammalian cells and kill bacteria $(8,21)$. As a result, pyroptosis was redefined as gasdermin-mediated programmed necrotic cell death (22).

Caspase is a family of cysteine proteases that are expressed as zymogens activated by protease cleavage $(23,24)$. The mature enzymes predominantly cleave at P1-site aspartate residues within their substrates and play crucial roles in cell death, tissue remodeling, and differentiation (25-27). Caspases can be divided

\section{Significance \\ The inflammasomes are signaling platforms that promote the activation of inflammatory caspases such as caspases-1, $-4,-5$, and -11 , which cleave gasdermin D (GSDMD) to induce pyroptotic cell death. The mechanisms of GSDMD recognition by inflammatory caspases remain poorly understood. Here, we demonstrate that the catalytic domains of inflammatory caspases can directly bind to GSDMD or its cleavage site peptide, FLTD. A GSDMD-derived inhibitor, $\mathrm{N}$-acetyl-Phe-Leu-Thr-Asp-chloromethylketone (Ac-FLTD- CMK), inhibits GSDMD cleavage in vitro and suppresses pyrop- tosis downstream of both canonical and noncanonical inflam- masomes. By contrast, the inhibitor does not target caspase-3 or apoptosis, suggesting that it is specific for inflammatory caspases. Structure of caspase-1 in complex with Ac-FLTD-CMK reveals ex- tensive enzyme-inhibitor interactions that shed light on GSDMD recognition by inflammatory caspases.}

Author contributions: J.Y., Z.L., C.W., J.K.R., D.W.A., and T.S.X. designed research; J.Y. Z.L., C.W., R.Y., J.K.R., O.W.P., W.S., and Y.C. performed research; J.K.R., O.W.P., and D.W.A. designed and performed the coimmunoprecipitation and CRISPR knockout studies; G.R.D. contributed new reagents/analytic tools; J.Y., Z.L., C.W., R.Y., J.K.R., W.S., Y.C., G.R.D., D.W.A., and T.S.X. analyzed data; and J.Y. and T.S.X. wrote the paper.

The authors declare no conflict of interest.

This article is a PNAS Direct Submission.

Published under the PNAS license.

Data deposition: The atomic coordinates and structure factors have been deposited in the Protein Data Bank, www.wwpdb.org (PDB ID code 6BZ9).

${ }^{1}$ To whom correspondence should be addressed. Email: tsx@case.edu.

This article contains supporting information online at www.pnas.org/lookup/suppl/doi:10 1073/pnas.1800562115/-/DCSupplemental.

Published online June 11, 2018. 
A

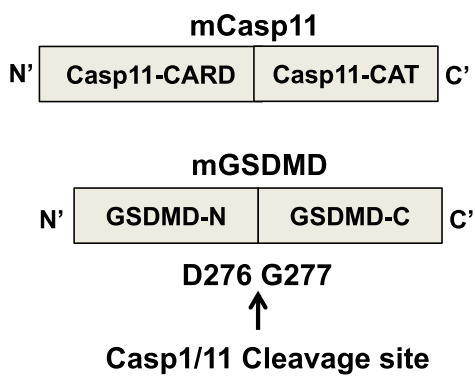

B

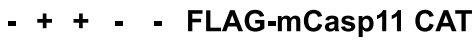

- $\ldots++$ FLAG-mCasp11

C254A CAT

+ - + + Myc-mGSDMD

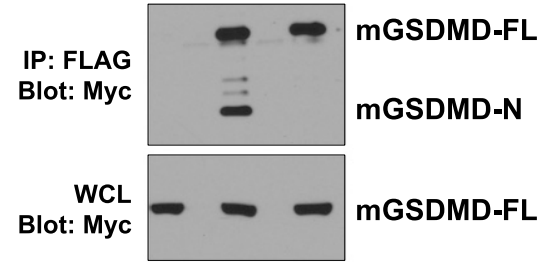

$\begin{aligned} \text { IP: FLAG } & \\ \text { Blot: FLAG } & \text { mCasp11 }\end{aligned}$

$\mathrm{D}$
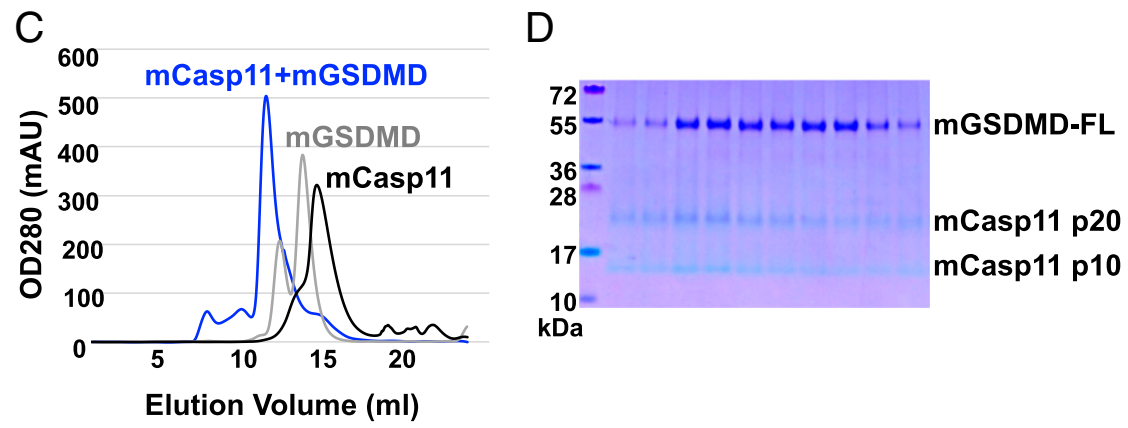

Fig. 1. Interaction between GSDMD and inflammatory caspases. (A) Domain structures of caspase-11 (Casp11) and GSDMD marked with its cleavage site by inflammatory caspases [murine (m)GSDMD numbering]. (B) WT or C254A mutant of FLAG tagged caspases-11 catalytic domain (FLAG-mCasp11 CAT) was cotransfected with Myc-tagged mGSDMD in HEK293T cells. After $24 \mathrm{~h}$, cell lysates were immunoprecipitated (IP) with anti-FLAG antibody and blotted with either anti-FLAG or anti-Myc antibodies. (C) Size-exclusion chromatograms for purified recombinant murine caspase-11 catalytic domain (C254A) (black), fulllength mGSDMD (gray), and caspase-11-GSDMD mixture (blue). (D) SDS/PAGE gel is shown for peak fractions from the blue chromatogram in $C$.

into inflammatory caspases (such as caspases-1, $-4,-5$, and -12 in humans and caspases-1, -11 , and -12 in mice) and apoptotic caspases (such as caspases-2, -3, -6, -7, -8, -9, and -10) (28). Several caspase inhibitors have been developed and are being widely used in the field. For example, $N$-benzyloxycarbonyl-ValAla-Asp(O-Me)-fluoromethylketone (z-VAD-FMK) is a pancaspase inhibitor that targets most caspases $(29,30)$; peptide YVAD-based inhibitor is specific for caspase- 1 but is 200- to 500 -fold less potent for caspases-4 and -5 (30, 31); peptide DEVE-based inhibitor is specific for caspase-3 (30, 31). Currently, no inhibitor that specifically targets all inflammatory caspases has been reported.

Each of the caspases has its own set of physiological substrates $(27,30,31)$. Some caspases (such as caspases-1, $-2,-3,-6,-7$, and -8) recognize a variety of substrates, while others (such as caspases-4, -5 , and -11) recognize a limited number of substrates. Even though caspases- $1,-4,-5$, and -11 all cleave GSDMD, only caspase-1 cleaves pro-IL-1 $\beta(4,17)$. The molecular mechanisms of such substrate specificity are poorly understood. In the present study, we investigated the molecular mechanisms of GSDMD recognition and cleavage by inflammatory caspases, a crucial triggering event in pyroptosis and inflammatory cytokine secretion. We show that inflammatory caspases directly bind to GSDMD as well as the cleavage site peptide, FLTD. Building on this knowledge, we designed an inhibitor specific for inflammatory caspases, $\mathrm{N}$ acetyl-Phe-Leu-Thr-Asp-chloromethylketone (Ac-FLTD-CMK). It is potent against caspases- $1,-4,-5$, and -11 , but not the apoptotic caspases such as caspase- 3 , in both biochemical and cellular assays. Furthermore, crystal structure of caspase- 1 in complex with Ac-FLTD-CMK demonstrates that both hydrophobic and hydrophilic interactions contribute to the association of the inhibitor with caspase-1. Together, this study furthers our understanding of GSDMD recognition by inflammatory caspases and reports a GSDMD-derived covalent inhibitor specific for inflammatory caspases, which can serve as a tool for investigating inflammasome signaling.

A

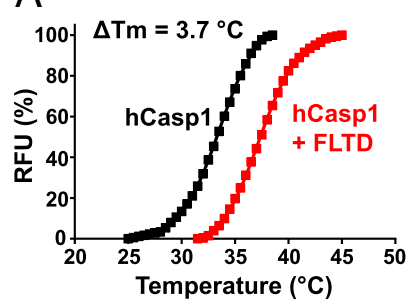

B
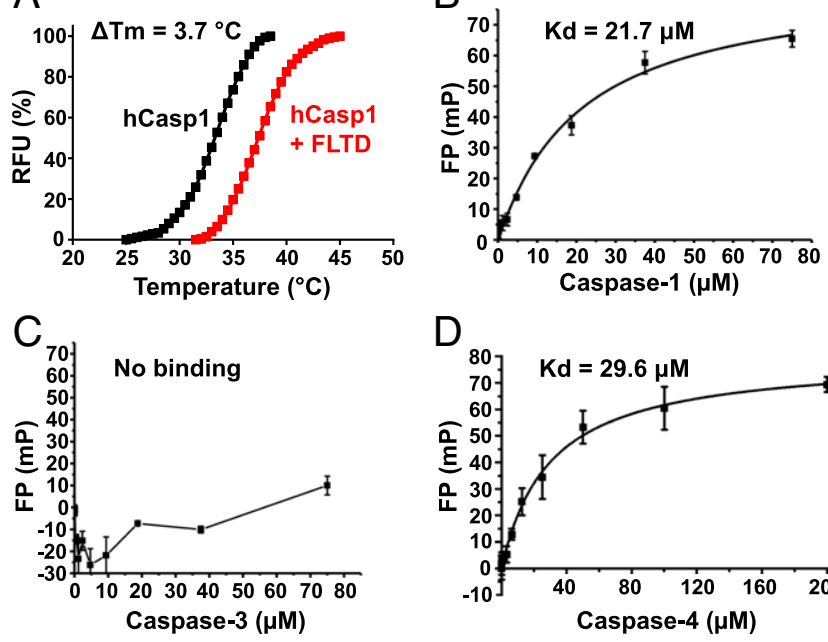

$\mathrm{D}$

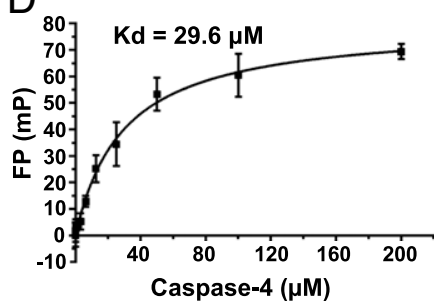

Fig. 2. The GSDMD cleavage site peptide FLTD directly binds caspase-1. (A) Thermal shift assay between the FLTD peptide and the human caspase-1 (hCasp1) catalytic domain (C285A). The melting temperature $(\mathrm{Tm})$ has a $3.7^{\circ} \mathrm{C}$ shift in the presence of FLTD peptide (shown in red) compared with caspase-1 with DMSO control (shown in black). (B-D) Fluorescence polarization (FP) assays for binding of FAM-Ahx-FLTD to human caspase-1 catalytic domain (C285A) (B), human caspase-3 catalytic domain (C163A) (C), and human caspase-4 catalytic domain (C258A) (D). Data shown are representative of mean \pm SD of at least three independent experiments. 
A

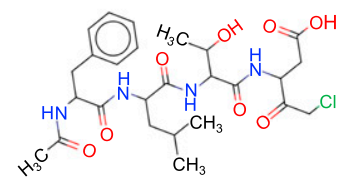

C
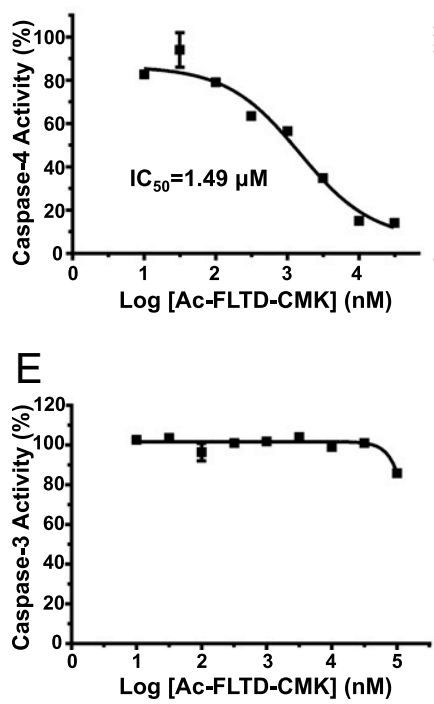

Fig. 3. AC-FLTD-CMK specifically inhibits inflammatory caspases. $(A)$ Chemical structure of the GSDMD-derived peptide inhibitor Ac-FLTD-CMK. (B-D) Enzymatic activities of human caspase- $1(B)$, caspase- $4(C)$, caspase- $5(D)$, and caspase$3(E)$ in the presence of AC-FLTD-CMK. Data shown are representative of mean \pm SD of at least three independent experiments.

\section{Results}

Inflammatory Caspases Directly Bind GSDMD. To investigate the interaction between caspases and GSDMD, FLAG-tagged catalytic domain of murine caspase-11 (FLAG-mCasp11 CAT) and Myc-tagged full-length murine GSDMD (Myc-mGSDMD) were coexpressed in HEK-293T cells (Fig. 1). The full-length GSDMD was coimmunoprecipitated by both wild-type (WT) and catalytically inactive (C254A) caspase-11, with GSDMD partially processed by the former (Fig. $1 B$ ). By contrast, no obvious binding was detected between the caspase-11 CARD and GSDMD (SI Appendix, Fig. S1 $A$ ), suggesting that the catalytic domain of caspase11 is sufficient for binding to GSDMD. To test the direct association between inflammatory caspases and GSDMD, recombinant proteins for murine caspase-11 catalytic domain (C254A) or human caspase-1 catalytic domain (C285A) was incubated with murine or human full-length GSDMD, followed by analysis with size-exclusive chromatography. The catalytically inactive mutant caspases were used to prevent cleavage of and dissociation from GSDMD. When purified full-length GSDMD protein and caspases were mixed together, the appearance of a larger protein complex containing both GSDMD and caspases- 1 and -11 was observed (Fig. $1 C$ and $D$ and SI Appendix, Fig. S1 $B$ and $C$ ), suggesting direct association of inflammatory caspases with GSDMD.

GSDMD Cleavage Site Peptide and Its Derivative Bind Inflammatory Caspases. Since GSDMD directly associates with caspase-1 and caspase-11, we hypothesized that peptides containing the GSDMD cleavage site residues FLTD (SI Appendix, Fig. S2A) may participate in caspase binding. A thermal shift assay was used to examine the binding between caspase- 1 and the FLTD peptide. A $3.7^{\circ} \mathrm{C}$ shift of the melting temperature for the human caspase- $1 \mathrm{C} 285 \mathrm{~A}$ mutant was observed in the presence of the peptide, indicating that the cleavage site peptide is sufficient for caspase-1 binding (Fig. 2A). To quantify this binding, we performed fluorescence polarization assays between caspases and an FLTD peptide containing N-terminal fluorescent label carboxyfluorescein (FAM), followed by an aminohexanoic acid (Ahx) spacer. The binding affinities of FAM-AhxFLTD to human caspase-1 (C285A) and to human caspase-4 (C258A) are $21.7 \mu \mathrm{M}$ and $29.6 \mu \mathrm{M}$, respectively, compared with no significant binding to human caspase-3 (C163A) within the range of concentrations tested (Fig. 2 and SI Appendix, Fig. S2 B and C). This direct binding is further confirmed by Biacore analysis of the peptide association with human caspase-1 (C285A) (SI Appendix, Fig. S2D). Collectively, our data suggest direct and specific binding between the FLTD peptide and inflammatory caspases, in agreement with previous findings that GSDMD is cleaved by inflammatory, rather than apoptotic, caspases (5).

A GSDMD-Derived Inhibitor Specifically Targets Inflammatory, but Not Apoptotic, Caspases. Based on the specific binding of the FLTD peptide to inflammatory caspases and on previous studies of caspase inhibitors using halomethyl ketone groups to covalently modify the catalytic site Cys residue (31), the GSDMD-derived peptide inhibitor Ac-FLTD-CMK (Fig. 3A) was designed to target inflammatory caspases. To investigate the effects of Ac-FLTD-CMK on caspase activities, we performed in vitro caspase activity assays using synthetic caspases-1, -4 , and -5 substrate $N$-acetyl-Trp-GluHis-Asp-7-amido-4-methylcoumarin (Ac-WEHD-AMC, Enzo Life Sciences) and caspase-3 substrate Ac-DEVD-AMC. Ac-FLTDCMK potently inhibited the enzymatic activities of caspases-1, -4, and -5 , but not caspase-3 (Fig. $3 B-E$ and SI Appendix, Fig. $\mathrm{S} 3 A$ ). In addition to the synthetic caspase substrates, cleavage of the physiological substrate GSDMD was investigated in the presence of Ac-FLTD-CMK and z-VAD-FMK, a pan-caspase inhibitor. As expected, caspases-1, -4 , and -5 cleaved human
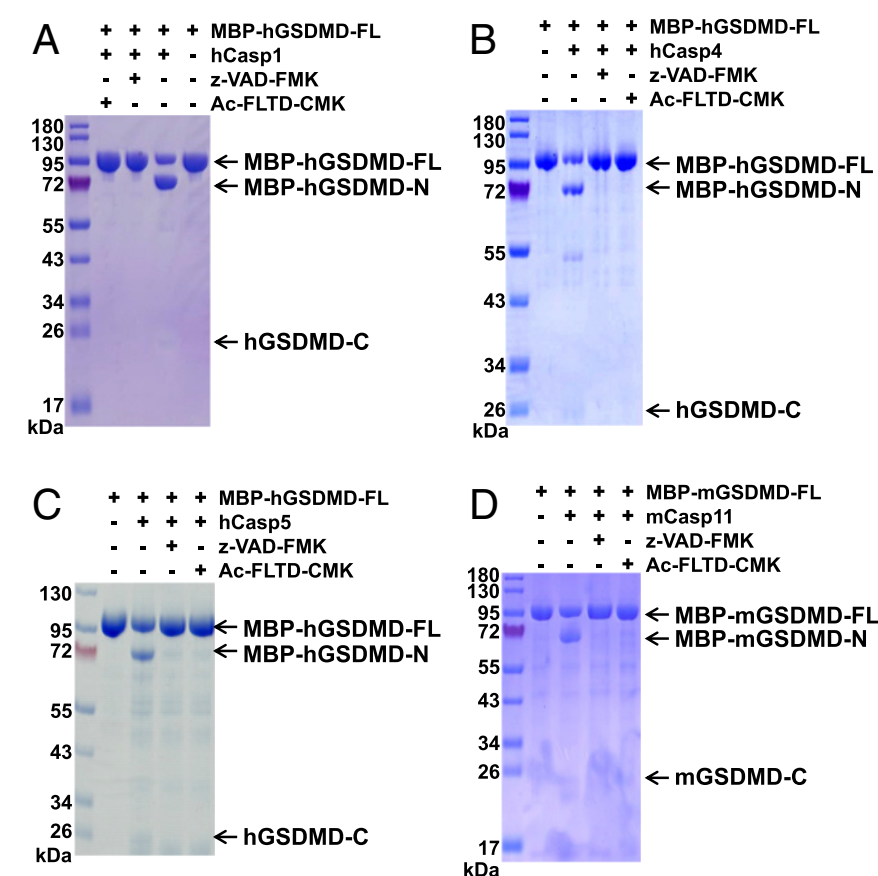

Fig. 4. AC-FLTD-CMK inhibits in vitro GSDMD cleavage by inflammatory caspases. Ac-FLTD-CMK inhibits MBP-hGSDMD cleavage by human caspase-1 (hCasp1) (A), hCasp4 (B), hCasp5 (C), and MBP-mGSDMD cleavage by murine (m)Casp11 (D) at $10 \mu \mathrm{M}$ concentration. 
A

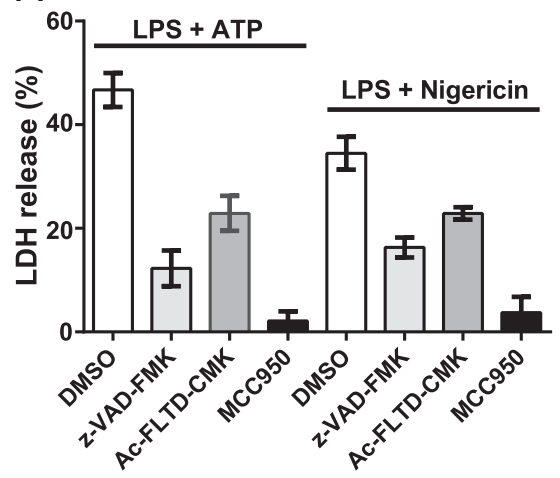

C

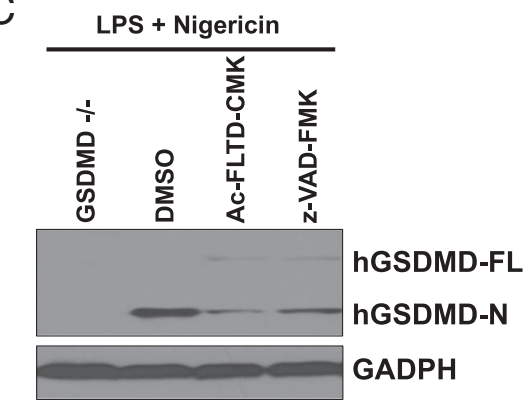

E

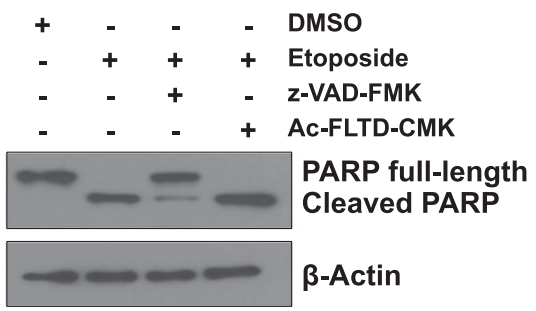

B

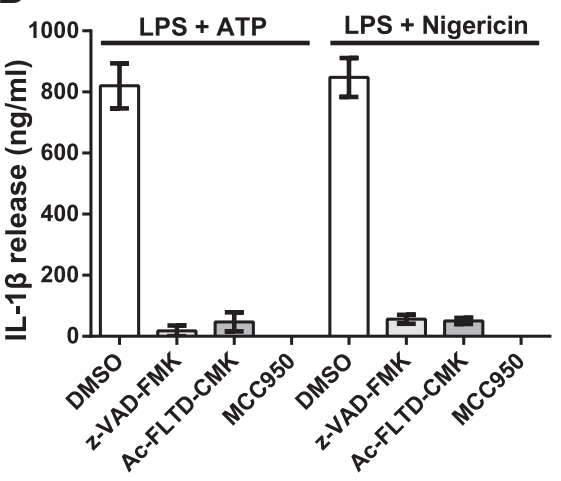

D

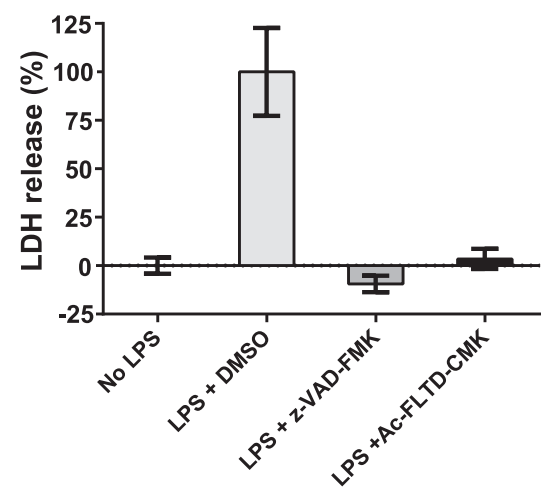

Fig. 5. Ac-FLTD-CMK inhibits pyroptotic cell death and IL-1 $\beta$ release, but not apoptosis. $(A$ and $B$ ) Bone marrow-derived macrophage cells were primed with $200 \mathrm{ng} / \mathrm{mL}$ LPS for $4 \mathrm{~h}$, followed by either $5 \mathrm{mM}$ ATP or $10 \mu \mathrm{M}$ nigericin treatment to activate the NLRP3 inflammasome. Z-VAD-FMK, Ac-FLTD-CMK, or MCC950 was added at $10 \mu \mathrm{M}$ concentration $30 \mathrm{~min}$ before ATP or nigericin treatment. Cytotoxicity was measured by lactate dehydrogenase (LDH) release assay $(A)$ and IL-1 $\beta$ release was determined by ELISA $(B)$. (C) THP-1 cells were treated with LPS, nigericin, and the indicated inhibitors at $10 \mu \mathrm{M}$ concentrations. GSDMD-knockout THP-1 cells were used as a control. Cleavage of GSDMD was analyzed through Western blot, with GAPDH as loading controls. (D) RAW 264.7 cells were primed with $200 \mathrm{ng} / \mathrm{mL}$ LPS for $4 \mathrm{~h}$ and then electroporated with LPS at $1 \mu \mathrm{g} / 10^{6}$ cells. z-VAD-FMK or Ac-FLTD-CMK was electroporated together with LPS at $10 \mu \mathrm{M}$ concentration. Cytotoxicity was measured by LDH release assay. $(E)$ Jurkat cells were treated with DMSO, $10 \mu \mathrm{M}$ z-VAD-FMK, or ACFLTD-CMK, followed by $10 \mu \mathrm{M}$ etoposide to induce apoptosis. PARP cleavage was detected as a read-out for apoptosis, with $\beta$-actin as loading controls. Data in $A, B$, and $D$ are representative of mean \pm SD of at least three independent experiments.

GSDMD and caspase-11 cleaved mouse GSDMD, which were inhibited by both Ac-FLTD-CMK and z-VAD-FMK (Fig. 4). Together, our data suggest that Ac-FLTD-CMK is an inhibitor that is specific for inflammatory caspases.

AC-FLTD-CMK Inhibits Pyroptosis and IL-1 $\beta$ Release, but Not Apoptosis. To probe the activities of Ac-FLTD-CMK in cells, we studied its effects on canonical and noncanonical inflammasome activation in macrophages. Both Ac-FLTD-CMK and z-VAD-FMK, as well as a known NLRP3 inhibitor, MCC950 (32), inhibited pyroptotic death of macrophages upon NLRP3 inflammasome activation (Fig. $5 A$ ) and IL-1 $\beta$ release (Fig. 5B). However, neither Ac-FLTD-CMK nor ZVAD-FMK inhibited the ASC speck formation in an immortalized murine bone marrow-derived macrophage cell line that stably expresses a cerulean-tagged ASC protein (33), in contrast to MCC950 (SI Appendix, Fig. S4). This suggests that both Ac-FLTD-CMK and zVAD-FMK target caspases, but not the upstream event of inflammasome assembly. In agreement, Ac-FLTD-CMK and z-VAD-FMK inhibited GSDMD cleavage upon NLRP3 inflammasome activation (Fig. 5C). To probe the effects of Ac-FLTD-CMK on noncanonical inflammasome activation, LPS was electroporated into RAW 264.7 cells, which induced pyroptotic cell death. This was suppressed by both Ac-FLTD-CMK and z-VAD-FMK (Fig. 5D), consistent with their effects on inflammatory caspases. Because Ac-FLTD-CMK did not inhibit caspase-3 (Fig. 3 and SI Appendix, Fig. S3), we tested whether it affects apoptosis. Apoptosis was induced in Jurkat cells with etoposide treatment. We observed that PARP cleavage (Fig. $5 E$ ), caspases-3 and -8 cleavage (SI Appendix, Fig. S3B), and cell death (SI Appendix, Fig. S3C) were drastically reduced by z-VADFMK, but not Ac-FLTD-CMK, treatment. Collectively, our data suggest that Ac-FLTD-CMK specifically inhibits inflammatory caspases, but not apoptotic caspases, at the cellular level.

Crystal Structure of Caspase-1 in Complex with Ac-FLTD-CMK. To reveal how Ac-FLTD-CMK engages inflammatory caspases, we determined a crystal structure of the catalytic domain of the 
A

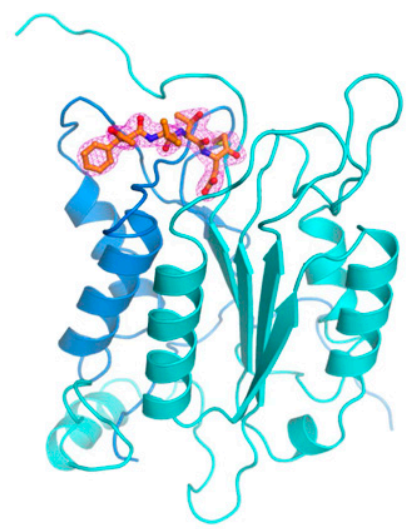

C

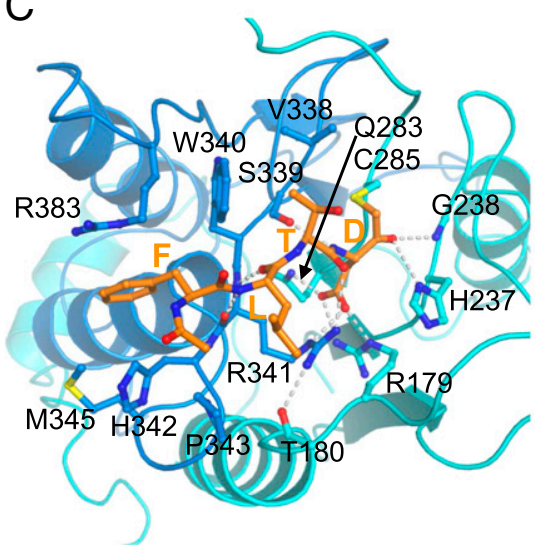

B
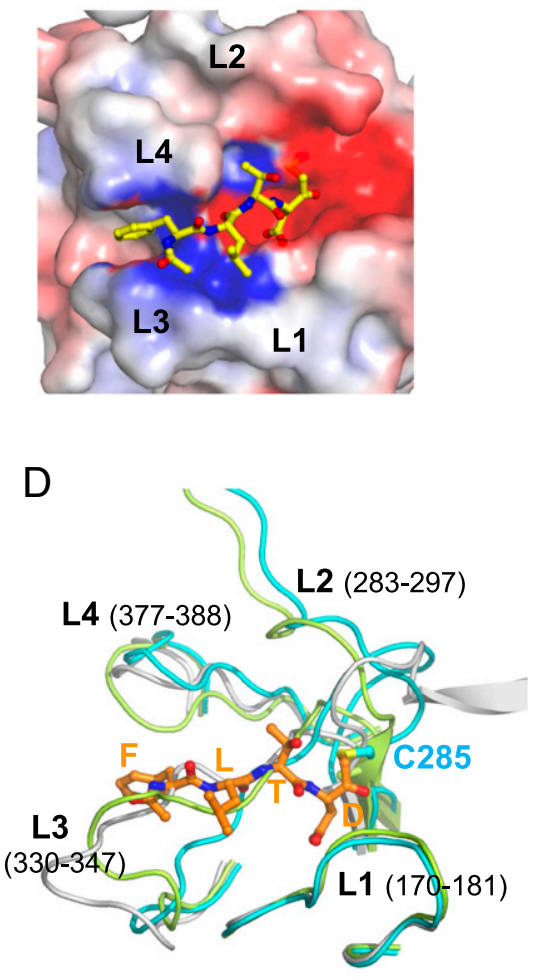

Fig. 6. Crystal structure of human caspase-1 in complex with Ac-FLTD-CMK. $(A)$ Overview of the crystal structure of human caspase-1 catalytic domain in complex with the Ac-FLTD-CMK inhibitor with the $2 F o-F c$ electron density map (magenta) contoured at $1 \sigma$. The p20 and p10 subunits of caspase-1 are colored cyan and marine, respectively, and the inhibitor is colored orange. $(B)$ The electrostatic charge surface of caspase-1 at its catalytic pocket is shown on a scale of $-5 \mathrm{kT} / \mathrm{e}$ (red) to $5 \mathrm{kT} / \mathrm{e}$ (blue), with the four catalytic site loops L1 to L4 marked. The bound Ac-FLTD-CMK is shown as a ball-and-stick model. (C) Detailed interaction between the inhibitor and caspase-1 is shown, with hydrogen bonds indicated as gray dotted lines. The inhibitor is shown in orange sticks. (D) Comparison of the four active-site loops from the caspase-1-Ac-FLTD complex (cyan), caspase-1 ligand free structure (green; PDB ID code 1SC1), and caspase-1 zymogen structure (gray; PDB ID code 3E4C).

human caspase-1 in complex with Ac-FLTD-CMK at $1.8-\AA$ resolution (PDB ID code 6BZ9). The crystallographic asymmetric unit contains a p20/p10 heterodimer bound to an AcFLTD-CMK inhibitor at the catalytic groove (Fig. $6 A$ ), cradled by L1 to L4 loops (Fig. 6B), similar to a previous structure of caspase-1 in complex with z-VAD-FMK (SI Appendix, Fig. S5A) (34). Both hydrophilic and hydrophobic interactions contribute to the extensive interaction between Ac-FLTD-CMK and caspase-1 (Fig. $6 C$ ), which buries $550 \AA^{2}$ of the total $820 \AA^{2}$ of solvent-accessible surface area for the inhibitor. Specifically, at the P1 site, the Asp residue from Ac-FLTD-CMK forms mainchain-main-chain hydrogen bonds with G238 and S339, a mainchain-side-chain hydrogen bond with $\mathrm{H} 237$, and side-chain hydrogen bonds with R179, Q283, and R341 from caspase-1. Such interaction is strengthened by a covalent bond formed by the CMK moiety and C285 from caspase-1. The P2-site Thr residue forms a main-chain-side-chain hydrogen bond with R341 and hydrophobic contacts with V338 and W340. The P3-site Leu residue forms two main-chain-main-chain hydrogen bonds with R341, resembling those in an antiparallel $\beta$ sheet. The side chain of the P4-site Phe residue forms extensive hydrophobic and van der Waals interactions with the side chains of W340, H342, M345, and R383. The N-terminal acetyl moiety in Ac-FLTD-CMK is close to $\mathrm{H} 342$ and P343, which may allow for additional hydrogen bonds and hydrophobic contacts with P5 residue in the full-length GSDMD protein. Superposition of our structure with a previously reported caspase-1 zymogen structure (PDB ID code 3E4C) (35) and a caspase- 1 structure in the absence of bound ligand (PDB ID code 1SC1) (36) demonstrates drastic conformational change at the four active-site loops, L1 to L4 (Fig. 6D), in agreement with previous analyses, revealing key roles of these loops in mediating the recognition of caspase substrates $(25,26)$.

Structure-based sequence alignment of inflammatory and apoptotic caspases reveals that the L1 and L2 loop residues engaging the inhibitor are identical among caspases-1, -3, -4, -5, and -11, whereas the L3 and L4 loop residues are less conserved, with the L3 loop harboring the most extensive interaction (Fig. $6 \mathrm{C}$ and $\mathrm{SI} A \mathrm{p}$ pendix, Fig. S5B). In fact, three of seven inhibitor-binding residues in the caspase-1 L3 loop (H342, P343, and M345) are not conserved among the different caspases, neither is the L4 loop residue R383. Since the M345 and R383 residues are involved in hydrophobic and van der Waals contact with the P4-site residue of the inhibitor (Fig. 6C), it is possible that the negatively charged Asp residues in caspase- 3 at these two equivalent positions may disrupt such interactions. As a result, divergent L3 and L4 loop residues may dictate the specific recognition of GSDMD by inflammatory, rather than apoptotic, caspases.

\section{Discussion}

Despite the identification of GSDMD as an effector of pyroptosis, it remains unclear how inflammatory caspases specifically recognize GSDMD, which leads to its cleavage and formation of membrane pores by its $\mathrm{N}$-domain. In the current study, we examined the interactions between inflammatory caspases and 
GSDMD as well as peptides containing the cleavage site residues FLTD. Our data suggest that GSDMD forms a stable complex with caspase-1 or caspase-11, which may be recapitulated by its cleavage site FLTD peptide. Taking advantage of this peptide, we designed a targeted covalent inhibitor Ac-FLTD-CMK. Our crystal structure of caspase-1 in complex with Ac-FLTD-CMK reveals extensive enzyme-inhibitor interactions which shed light on the mechanisms of GSDMD recognition by inflammatory caspases. Because such recognition is a crucial molecular event that triggers pyroptotic cell death and facilitates secretion of inflammatory cytokines, understanding the detailed molecular interplay is of fundamental importance to the study of innate immunity and inflammation.

In addition to GSDMD, caspase- 1 recognizes a number of other substrates, such as pro-IL-1 $\beta$ with a cleavage site of YVHD that closely resembles FLTD in GSDMD. However, pro-IL-1 $\beta$ is not cleaved by inflammatory caspases other than caspase- $1(4,17)$, suggesting that regions outside the cleavage site may contribute to specific substrate recognition by different inflammatory caspases as well. These regions may provide proper domain structure to productively present the cleavage site, or may furnish additional enzyme-substrate interactions at the so-called exosites (26), which was previously demonstrated for PARP1 recognition by caspase-7 (37). These mechanisms may thus underlie the different substrate specificities between caspase- 1 and caspases- $4,-5$, and -11 . The molecular details of such specific recognition await further investigation.

Currently there is no inhibitor that specifically targets the inflammatory caspase subset, which plays critical roles in canonical and noncanonical inflammasome signaling pathways. Here, we designed the FLTD peptide-based inhibitor Ac-FLTD-CMK using the GSDMD cleavage site sequence and taking advantage of previous reports of synthetic caspase inhibitors containing halomethyl ketone groups. The CMK moiety endows both potent

1. Martinon F, Burns K, Tschopp J (2002) The inflammasome: A molecular platform triggering activation of inflammatory caspases and processing of prolL-beta. $\mathrm{Mol} \mathrm{Ce} / \mathrm{l}$ 10:417-426.

2. Guo H, Callaway JB, Ting JPY (2015) Inflammasomes: Mechanism of action, role in disease, and therapeutics. Nat Med 21:677-687.

3. Latz E, Xiao TS, Stutz A (2013) Activation and regulation of the inflammasomes. Nat Rev Immunol 13:397-411.

4. Afonina IS, Müller C, Martin SJ, Beyaert R (2015) Proteolytic processing of interleukin 1 family cytokines: Variations on a common theme. Immunity 42:991-1004.

5. Shi J, et al. (2015) Cleavage of GSDMD by inflammatory caspases determines pyroptotic cell death. Nature 526:660-665.

6. Kayagaki $\mathrm{N}$, et al. (2015) Caspase-11 cleaves gasdermin D for non-canonical in flammasome signalling. Nature 526:666-671.

7. He W-T, et al. (2015) Gasdermin $D$ is an executor of pyroptosis and required for interleukin-1 $\beta$ secretion. Cell Res 25:1285-1298.

8. Ding J, et al. (2016) Pore-forming activity and structural autoinhibition of the gasdermin family. Nature 535:111-116.

9. Kuang S, et al. (2017) Structure insight of GSDMD reveals the basis of GSDMD au toinhibition in cell pyroptosis. Proc Natl Acad Sci USA 114:10642-10647.

10. Liu Z, et al. (2018) Structures of the gasdermin D C-terminal domains reveal mechanisms of autoinhibition. Structure 26:778-784.e3.

11. Bergsbaken T, Fink SL, Cookson BT (2009) Pyroptosis: Host cell death and inflammation. Nat Rev Microbiol 7:99-109.

12. Sborgi L, et al. (2016) GSDMD membrane pore formation constitutes the mechanism of pyroptotic cell death. EMBO J 35:1766-1778.

13. Aglietti RA, et al. (2016) GsdmD p30 elicited by caspase-11 during pyroptosis forms pores in membranes. Proc Natl Acad Sci USA 113:7858-7863.

14. Liu X, et al. (2016) Inflammasome-activated gasdermin D causes pyroptosis by forming membrane pores. Nature 535:153-158.

15. Chen X, et al. (2016) Pyroptosis is driven by non-selective gasdermin-D pore and its morphology is different from MLKL channel-mediated necroptosis. Cell Res 26 1007-1020.

16. Liu X, Lieberman J (2017) A mechanistic understanding of pyroptosis: The fiery death triggered by invasive infection. Adv Immunol 135:81-117.

17. Kayagaki N, et al. (2011) Non-canonical inflammasome activation targets caspase-11. Nature 479:117-121.

18. Kayagaki $\mathrm{N}$, et al. (2013) Noncanonical inflammasome activation by intracellular LPS independent of TLR4. Science 341:1246-1249. reactivity to the active site Cys residue and good membrane permeability compared with peptides linked to aldehyde groups (-CHO) (31). In agreement, the Ac-FLTD-CMK inhibitor targets inflammatory caspases, but not apoptotic caspase-3, in enzymatic analyses. Importantly, the inhibitor selectively suppresses canonical and noncanonical inflammasome pathways in our cellular assays, but not apoptosis, within the concentrations tested here. Clearly, further improvement of this inhibitor is required because the CMK group is highly reactive and could potentially target other cysteine proteases at elevated concentrations. Nonetheless, data presented here demonstrate that Ac-FLTD-CMK is a valuable tool reagent for studying the inflammasome signaling pathways.

\section{Materials and Methods}

Details are described in SI Appendix, SI Materials and Methods. This includes information on expression and purification of caspases-1, $-3,-4,-5$, and -11 , caspase-inhibitor/peptide binding assays, caspase activity analyses, assays for inflammasome activation and apoptosis, and crystal structure determination. The PDB ID code for this work is 6BZ9.

ACKNOWLEDGMENTS. We thank Dr. Quyen and Q. Hoang for kindly providing us with the caspase-1 expression constructs and Dr. Edward A. Miao for providing us with the caspases-4, -5 , and -11 CDNA. The cDNA libraries of human and murine macrophages were a gift from Bin Lin (National Institute of Allergy and Infectious Diseases, NIH). We thank Pengcheng Zhou for helping with procurement of AC-FLTD-CMK from KE BIOCHEM. We thank the members of the T.S.X., D.W.A., G.R.D., and Ramakrishnan laboratories for insightful discussions and comments. The X-ray diffraction data were collected at Automated Macromolecular Crystallography and Frontier Macromolecular Crystallography beamlines, Center for Synchrotron Biosciences, Brookhaven National Laboratory (Upton, NY). T.S.X. is supported by NIH Grants AR069908 and GM127609 and the Case Research Institute; Z.L. is supported by a postdoctoral fellowship from the American Association of Immunologists; J.K.R. is supported by NIH Grant T32 GM007250; and D.W.A. is funded by NIH Grants DK091222 and GM086550.

19. Hagar JA, Powell DA, Aachoui Y, Ernst RK, Miao EA (2013) Cytoplasmic LPS activates caspase-11: Implications in TLR4-independent endotoxic shock. Science 341: $1250-1253$

20. Shi J, et al. (2014) Inflammatory caspases are innate immune receptors for intracellular LPS. Nature 514:187-192.

21. Wang $Y$, et al. (2017) Chemotherapy drugs induce pyroptosis through caspase-3 cleavage of a gasdermin. Nature 547:99-103.

22. Shi J, Gao W, Shao F (2017) Pyroptosis: Gasdermin-mediated programmed necrotic cell death. Trends Biochem Sci 42:245-254.

23. Alnemri ES, et al. (1996) Human ICE/CED-3 protease nomenclature. Cell 87:171

24. Li J, Yuan J (2008) Caspases in apoptosis and beyond. Oncogene 27:6194-6206.

25. Shi $Y$ (2002) Mechanisms of caspase activation and inhibition during apoptosis. Mol Cell 9:459-470.

26. Fuentes-Prior P, Salvesen GS (2004) The protein structures that shape caspase activity, specificity, activation and inhibition. Biochem J 384:201-232.

27. Julien O, Wells JA (2017) Caspases and their substrates. Cell Death Differ 24: 1380-1389.

28. Jiménez Fernández D, Lamkanfi M (2015) Inflammatory caspases: Key regulators of inflammation and cell death. Biol Chem 396:193-203.

29. Slee EA, et al. (1996) Benzyloxycarbonyl-Val-Ala-Asp (OMe) fluoromethylketone (ZVAD.FMK) inhibits apoptosis by blocking the processing of (PP32. Biochem J 315: 21-24.

30. Garcia-Calvo M, et al. (1998) Inhibition of human caspases by peptide-based and macromolecular inhibitors. J Biol Chem 273:32608-32613.

31. Ekert PG, Silke J, Vaux DL (1999) Caspase inhibitors. Cell Death Differ 6:1081-1086

32. Coll RC, et al. (2015) A small-molecule inhibitor of the NLRP3 inflammasome for the treatment of inflammatory diseases. Nat Med 21:248-255.

33. Stutz A, Horvath GL, Monks BG, Latz E (2013) ASC speck formation as a readout for inflammasome activation. Methods Mol Biol 1040:91-101.

34. Scheer JM, Romanowski MJ, Wells JA (2006) A common allosteric site and mechanism in caspases. Proc Natl Acad Sci USA 103:7595-7600.

35. Elliott JM, Rouge L, Wiesmann C, Scheer JM (2009) Crystal structure of procaspase-1 zymogen domain reveals insight into inflammatory caspase autoactivation. J Biol Chem 284:6546-6553.

36. Romanowski MJ, Scheer JM, O'Brien T, McDowell RS (2004) Crystal structures of a ligand-free and malonate-bound human caspase-1: Implications for the mechanism of substrate binding. Structure 12:1361-1371.

37. Boucher D, Blais V, Denault J-B (2012) Caspase-7 uses an exosite to promote poly(ADP ribose) polymerase 1 proteolysis. Proc Natl Acad Sci USA 109:5669-5674. 